\title{
El papel de la Universidad en la disolución del distanciamiento entre la teoría y la práctica contable
}

\section{The role of higher education institutions in the estrangement between theory and practice in accounting}

\author{
Luz Estela Durán Caicedo*
}

luz.duran@unilibrecucuta.edu.co

Recibido: octubre 13 de 2015. Aceptado: noviembre 30 de 2015.

doi: 10.22209/rhs.v3n1.2a05

\section{Resumen}

El trabajo presenta como objetivo discutir la fisura existente entre la teoría y la práctica contable, enfatizando el papel que tienen las instituciones de educación superior en la formación de un profesional con competencias, para articular la teoría y la práctica contable. El estudio se fundamentó metodológicamente en la revisión de literatura especializada en la materia objeto de estudio. Los resultados de la investigación demuestran la necesidad de promover la aplicación de otros enfoques como la teoría crítica para el desarrollo de la investigación en ciencias contables, los cuales deben comenzar a considerar el problema vinculado con el distanciamiento entre la teoría y la práctica desde otra perspectiva distinta al positivismo. Asimismo, las nuevas tendencias en las que se presentan los fenómenos contables exigen la formación de un contador integral con competencias para que además de aplicar la norma, construya el conocimiento contable a partir de la integración de la teoría y la práctica.

Palabras clave: ciencia contable, práctica contable, fisura, investigación, universidad, contador público.

\begin{abstract}
The goal of this paper is to discuss the flaws there exist between theory and practice in accounting, emphasizing the role played by higher education institutions in training a professional with skills to articulate theory and practice. The discussion was based on literature review of the subject under study. The result of this investigation shows the need to promote others approaches such that the critical theory of the development of research in accounting sciences, in which starting to consider the problem linked with the estrangement between theory and practice must be considered from a different perspective than that of the positivism. Likewise, the new trends of accounting sciences demand the education of an integral accountant with skills to apply the norm and build the accounting knowledge from the integration of the theory and the practice.
\end{abstract}

Key Words: countable ciences, countable practice, fissure, investigation, university, public accountant.

\footnotetext{
Para citar este artículo: Durán Caicedo, L.E. (2015). El papel de la Universidad en la disolución del distanciamiento entre la teoría y la práctica contable. Rev Humanismo y Sociedad, 3(1-2), 35-42. doi: 10.22209/rhs.v3n1.2a05

*Docente Universidad Libre Seccional Cúcuta - Facultad de Contaduría. MSc. Prácticas Pedagógicas Universidad Francisco de Paula Santander. Especialista en Revisoría Fiscal Universidad Libre Seccional Cúcuta - Facultad de Contaduría. Doctorante en Ciencias Contables Universidad de los Andes ULA - Mérida Estado Táchira-Venezuela.
} 


\section{Introducción}

A continuación se presenta una reflexión sobre la fisura existente entre la teoría y la práctica contable, enfatizando en el papel que tienen las instituciones universitarias en la formación de un profesional con competencias para integrar adecuadamente la teoría y la práctica contable.

El trabajo se fundamentó en la revisión documental, para abordar de manera lógica y sistemática las propuestas teóricas relacionadas con la teoría y la práctica contable, así como el papel de las instituciones universitarias en la formación de un contador público con competencias para desenvolverse eficientemente en el escenario actual caracterizado por la globalización e internacionalización de los mercados, la estandarización de la normativa contable, entre otros aspectos.

Los resultados demuestran la necesidad de comenzar a desarrollar investigaciones en el área contable, donde se integren de manera sistemática la teoría y la práctica, pues en este campo, como lo sustentan los especialistas del área, se ha desarrollado primero la práctica $\mathrm{y}$, en función de esta se han realizado los estudios para generar los desarrollos teóricos que sustentan la disciplina contable.

Tal situación demuestra la necesidad de comenzar a formar un nuevo profesional en el área contable, con criterios de ética crítico y reflexivo, con competencias que le permitan además de aplicar la norma en la práctica, producir el conocimiento contable que la sociedad demanda, a partir de la integración de la teoría y la práctica contable, así como de la consideración de los fenómenos del contexto donde se desarrolla el hecho contable.

En este contexto, el trabajo se estructura en cuatro secciones: en la primera, se presenta una revisión de los fundamentos epistémicos de la ciencia contable; en la segunda, se aborda el distanciamiento entre la teoría y la práctica contable; en la tercera, se expone el rol de la Universidad en la formación del contador público; y en la cuarta, se argumentan algunas reflexiones a manera de conclusiones.

\section{Los fundamentos epistémicos de la disciplina contable}

En el devenir de la historia de la ciencia, se han presentado disputas relacionadas con los criterios que debe reunir un conocimiento para ser considerado científico. Concretamente en el campo contable, se ha desarrollado toda una discusión acerca del carácter científico de la contabilidad, encontrándose que algunos autores discrepan de su carácter científico, mientras que otros sostienen posiciones donde se fundamenta la cientificidad de la misma.

Al analizar la evolución del pensamiento contable Casal y Viloria (2007, p. 21), plantean que tradicionalmente la contabilidad

ha respondido fielmente a los paradigmas creados por el positivismo, a saber: el funcionalismo y el estructuralismo. Estos enfoques paradigmáticos le han dado la fisonomía que hoy tienen los sistemas contables, la clasificación y distribución de las cuentas en los estados financieros, las técnicas de registro utilizadas, el conjunto de tareas y tiempo del ciclo contable, los métodos de auditoria, las funciones de costos y utilidades, entre otros.

Al respecto, se destaca que la aplicación del enfoque positivista en la disciplina contable se encuentra en las posiciones de algunos autores, entre los que sobresalen Edwars y Bell (1961), Tua (1983), Mattessich (1995) y Thietart et al. (2003), los cuales han sido referenciados por Gómez (2003), al precisar que estas posturas se han sustentado en la utilización de teorías de corte positivista provenientes de otros campos disciplinares, como mecanismo para probar hipótesis instrumentales, pues a partir de observaciones particulares se han intentado explicar tanto la técnicas, procedimientos, normas y regulaciones contables, como el desarrollo empresarial. El sustento teórico de esta disciplina se ha fundamentado en

la aplicación de la teoría clásica de la organización, la teoría neoclásica de la economía, los desarrollos de la nueva economía institucional y en general los desarrollos ortodoxos de la microeconomía a la contabilidad de empresa (...) También es común la adopción de varias de las teorías conductistas en psicología y algunas vertientes del funcionalismo sociológico. La aplicación de métodos econométricos 
y estadísticos es la base de sus investigaciones (...) Por tanto, la perspectiva positiva de la economía y de otras ciencias sociales es, en general, el elemento más utilizado en este enfoque (p. 114).

Sobre la base del enfoque mencionado, se ha sustentado el desarrollo de los métodos y técnicas empleados en la elaboración de los sistemas contables, la clasificación y distribución de las cuentas en los estados financieros, la aplicación de los principios básicos de la contabilidad, la auditoría, las funciones de costo, inventario $\mathrm{y}$, cálculo de utilidades, entre otras actividades contables, pues como lo afirman Casal y Viloria (2007)

El método fundamental de la contabilidad ha sido la cuantificación de todas las magnitudes contables, en detrimento de la cualificación o explicación amplia y profunda. Todo ello producto de la objetivización positivista.

Como resultado de la incidencia de estos enfoques filosóficos y epistemológicos en la ciencia contable, su estado del arte o del conocimiento hasta mediados de la última década del siglo XX, era el de un oficio o artesanía contable, que la reducía al rol de técnica y no de ciencia, por lo que parecía empobrecida frente a otras ciencias que ocupaban sus espacios naturales. El ejercicio profesional era lo fundamental (p. 21).

En relación con los enfoques paradigmáticos aplicados en el campo contable, resulta de interés enfatizar que los investigadores han reflexionado ampliamente sobre algunos aspectos vinculados con la evolución histórica de la contabilidad, para precisar los elementos que configuran su naturaleza científica, los métodos utilizados para la construcción de conocimiento contable, así como los mecanismos orientados hacia la formalización de las construcciones teóricas generadas.

Siguiendo a Mattessich (2003) la contabilidad es una ciencia aplicada. En consecuencia, no puede abordarse ni desde un enfoque netamente positivista, ni desde una postura netamente crítico cultural «sino que debe estar basada en pensamiento racional y científico. Si falta esta exigencia, ni las cuestiones de la contabilidad ni los enormes problemas en la sociedad y el medio ambiente podrán resolverse» (p.119).
Para Tua (1995) si se limita el concepto de ciencia a la utilización de un instrumental positivista, estrictamente cognoscitivo, relegaremos los dos restantes tipos de conocimientos al ámbito metacientífico, con la que las construcciones normativas, típicas de la interpretación teleológica, no tendrían cabida en el concepto de ciencia, (...) En este caso, la contabilidad tendría poco de ciencia -la teoría general-y mucho de técnica -la construcción de sistemas contables-en la medida de que sus principales manifestaciones aparecen como aplicaciones ( $p .46)$.

No obstante, si el concepto de ciencia se asume desde una posición más amplia, se incluyen dentro del campo de lo científico los aspectos normativos e instrumentales y, en consecuencia, como se abarcan tanto las posturas normativas como positivas «la contabilidad, al igual que otras ciencias sociales, sería una disciplina científica» (Tua, 1995, p. 46).

Otra posición en torno a la disciplina contable la presenta Larrinaga (1999) al plantear que la utilización del enfoque crítico en la disciplina contable, a diferencia del positivismo, "presupone que no existe ningún hecho que sea independiente de teorías $\mathrm{y}$, por lo tanto, no se puede probar o rechazar ninguna teoría. En cambio, la verdad puede encontrarse en los procesos sociales e históricos» (p. 118). De allí, que los estudios contables fundamentados en la teoría crítica abordan la contabilidad considerando los fenómenos sociales, políticos y económicos, trascendiendo la distinción entre contabilidad financiera y de gestión «para estudiar simplemente la contabilidad» (Chua, citado por Larrinaga, p. 118).

\section{El distanciamiento entre el discurso científico y la práctica contable}

Existe un distanciamiento entre el discurso científico y la práctica contable. En palabras de Ospina (2005) esta divergencia se presenta

por una falta de compromiso de cada lado por interiorizar el otro, y por cimentar -cada lado negando su oposición, es decir, concretando una sola construcción. Partiendo de la negación de la dicotomía teoría práctica, asumiendo más bien una relación recíproca, yo diría, recursiva, no puede pensarse apriorísticamente un complejo teórico universal 
para orientar las prácticas sin nutrirse de estas, ya sea como apología de lo instrumental o como crítica orientadora de nuevas praxis al detectar las inconsistencias de las vigentes ( $p .84)$.

Dentro de este marco, Gómez (2006) expone la necesidad de precisar los aspectos que comprenden la teoría y la práctica en el ámbito contable, pues en la ciencia la teoría trasciende el campo vinculado con las definiciones formales o descripciones de las prácticas, en la búsqueda de proveer los modelos que intentan explicar, interpretar y comprender el comportamiento de la realidad objeto de estudio.

En el contexto de esta distinción entre la teoría y la práctica contable, destaca que el conocimiento científico en este ámbito, se orienta hacia la formulación de construcciones que intenten dar respuesta a los problemas de la sociedad; mientras que la norma contable se formula para intentar regular las relaciones entre las personas, y «la mayoría de veces desconoce el desarrollo operado en la ciencia y la técnica», tal es el caso de algunas leyes o decretos promulgados por las instituciones gubernamentales que promueven el desarrollo de un país, lo cual se evidencia «en nuestro saber principal: la disciplina contable y sus desarrollos se ven cercenados por la legislación profesional que aparece con bastante facilidad» (González, 2005, p. 171).

En la búsqueda de avanzar en la disolución del distanciamiento entre la teoría y la práctica contable, en el campo investigativo se ha propuesto la utilización de la teoría crítica como un enfoque alternativo para el estudio y abordaje de la realidad contable, en vista de que las formulaciones teóricas en este ámbito deben fundamentarse en una postura que permita desarrollar la crítica constante de las teorías y, su contrastación en el campo empírico por medio de la práctica, lo cual contribuye de manera determinante con la integración de la teoría y la práctica contable.

Desde la teoría interpretativa, los términos explicación, predicción y control, son sustituidos por comprensión, significado y acción. Por tanto, el objetivo de la investigación interpretativa no se centra en encontrar explicaciones causales de la vida social, sino en «revelar el significado de las formas particulares de la vida social mediante la articulación sistemática de las estructuras de significado subjetivo que rigen las maneras de actuar de los individuos» (Carr \& Kemmis, 1988, p.105). Bajo esta postura, la realidad es una construcción social que no puede conocerse «con independencia de quien quiera conocerla» (Carr \& Kemmis, 1988, p.116).

De otro lado, en el campo concreto de la práctica contable, Tua (1995) expone que en este ámbito las prácticas se han adoptado sin un fundamento teórico, pues la metodología empleada por la American Institute of Certified Public Accountants -Instituto Superior Americano de Contables- (AICPA) para la formulación de los Principios de Contabilidad

se basó principalmente en argumentos prácticos y no teóricos: se estudiaron procedimientos o técnicas específicas y se recomendaron ciertos procedimientos a base de su utilidad sin dependencia de una armazón general de teoría de la Contabilidad omnicomprensivo, y sin reconocer la necesidad de interdependencia de los varios procedimientos recomendados en diferentes esferas ( $p .43$ ).

Asimismo, el autor destaca la posición asumida por el Financial Accounting Standards Board, al plantear que «no creemos que el comité deba acometer un amplio programa relativo a los conceptos básicos de la contabilidad, sino que estimamos que este tipo de investigaciones debe de ser confiado al ámbito académico» (AICPA; citado por Tua, 1995, p. 43).

En este escenario, Laughlin (2011) plantea que el distanciamiento entre la teoría y la práctica contable se ha presentado, en vista de que los componentes de la profesión contable entendidos como la política (lo normativo), la investigación (realizada en las universidades) y la práctica (ejercicio profesional), se han abordado como tres mundos separados sin ninguna articulación o conexión. Para el autor, la política constituye el elemento que se ubica entre la investigación y la práctica contable.

Hasta ahora en el campo contable la práctica se ha abordado separadamente de la investigación realizada en las universidades, sin embargo, esta no puede desligarse del componente político. Siguiendo a Laughlin (2011), tanto la política como la investigación resultan relevantes para el desarrollo de la práctica. Por ende, los tres mundos (política, investigación y práctica) deben articularse a través del discurso, en la búsqueda de que la profesión contable pueda continuar prestando el servicio que la sociedad demanda en el contexto global. 
Al respecto Tucker y Parker (2014) plantean la necesidad de establecer un vínculo entre la investigación y la práctica, lo cual resulta pertinente tanto para los académicos como para los profesionales. Para los académicos a partir de esta relación se legitiman las actividades académicas, pues se abordan y se proponen soluciones para los problemas que se presentan en la práctica; mientras que para los profesionales la investigación contribuye con el mejoramiento de la toma de decisiones, así como con el desarrollo de nuevas prácticas que satisfagan las necesidades de la organización.

Otra posición al respecto la plantea González (2005) al exponer que la eliminación del distanciamiento entre la teoría y la práctica contable debe fundamentarse en una postura sociológica, que promueva el desarrollo de una práctica con capacidad para incidir y orientar las formulaciones teóricas y viceversa, lo cual implica un proceso constante y sistematizado de reflexión crítica de los fundamentos científicos «en la pretensión de iniciar creativos procesos de avance tanto de teorías como de prácticas contables con mayor calidad para acercar el razonamiento epistemológico a la práctica profesional» (p. 175).

En consecuencia, se requiere promover el desarrollo de investigaciones orientadas hacia la generación de construcciones teóricas, que fundamenten la aplicación de los principios y normativas contables en la práctica, pues como lo afirma Viloria (2013), pareciera que la investigación realizada por los Organismos Internacionales (IASB) que emiten la norma, es más fructífera en cuanto a la cantidad de los estándares contables construidos.

Como muestra de esta situación, destaca que cada año se revisan e incluyen nuevas normas e interpretaciones para orientar la práctica profesional. Sin embargo, también destaca que tal normativa, en algunos casos presenta

vacíos, inconsistencias y contradicciones, que generan conflictos en la interpretación y aplicación, creándose un ciclo sin fin, ante cada problema puntual, corregir la norma, pero, al hacerlo, se producen nuevos conflictos [Macintosh, 2009; citado por Viloria, 2013]. No obstante, la investigación universitaria, aunque más pausada, cumple, por una parte, con el rol de estudiar los aspectos problemáticos desde las teorías $y$, por la otra, de advertir a los organismos emisores de los problemas epistémicos (p. 50).

\section{El papel de la universidad en la formación del contador público ante las tendencias de las ciencias contables}

En el actual contexto donde se desarrollan las ciencias contables, la Universidad a partir de la docencia, investigación y extensión, tiene un papel preponderante en la formación de un profesional integral, crítico, reflexivo y autónomo, con competencias para construir conocimiento a partir de la articulación de la teoría y la práctica.

Concretamente en lo que respecta a la formación del contador, Tua (1993) expresa que existen dos mecanismos utilizados en la enseñanza de la contabilidad:

A. El primero, se orienta a trasmitir a los estudiantes los procedimientos y normas contables, como por ejemplo los principios de contabilidad generalmente aceptados, las Normas Internacionales de Información Financiera, o un plan de cuentas, para «desgranar sus recovecos, analizando su mecánica e ilustrando las cuestiones con ejemplos prácticos» (Tua, 1993, p. 44).

Bajo este mecanismo, el alumno aprende las técnicas de la contabilidad, pero no aprende los fundamentos de las ciencias contables. Se forma un profesional para aplicar la norma en el campo empírico, con la limitación de que desconoce los fundamentos que sustentaron la formulación de la norma, así como su vinculación con la epistemología contable. «Incluso, corre el peligro de confundir la teoría general de la contabilidad con el conjunto de normas que, dictadas por los correspondientes organismos competentes, gobiernan la práctica contable» (Tua, 1993,p. 44).

Este mecanismo, ha prevalecido en la educación universitaria tradicional. No obstante, cabe precisar que un profesional formado bajo la concepción educativa descrita, no se encontrará formado con las competencias requeridas para enfrentar adecuadamente los cambios que se han presentado en la disciplina contable.

B. El segundo, se fundamenta en la aplicación de nuevos enfoques educativos para el desarrollo del proceso de enseñanza - aprendizaje, como el de competencias, el cual se orienta hacia la formación de profesionales críticos y autónomos, con competencias para construir conocimiento contable a partir de la integración de la teoría y la práctica. Tal enfoque, implica una formación 
integral del docente para concebir la disciplina contable como un conjunto de fundamentos epistémicos aplicados a un caso determinado para obtener una norma. Bajo esta concepción, la enseñanza de la contabilidad debe partir de «las nociones básicas de teoría del conocimiento, ya que en los diferentes roles que este profesional asume, se encuentra el de docente formador de nuevos conocimientos» (Tua, 1993, p. 44).

Sobre la base de lo expuesto se deduce que un profesional integral en el área contable debe tener competencias para el dominio de la disciplina, sus bases teóricas, así como las prácticas de la misma, pues la enseñanza de la contabilidad no debe limitarse solo a la teoría contable, pero tampoco a la aplicación de la práctica sin un fundamento teórico estructurado y sistematizado.

Por ende, la enseñanza de la disciplina contable debe partir del análisis y discusión crítica de los fundamentos teóricos que sustentan y estructuran la práctica contable, lo cual implica un replanteamiento curricular, aunado a una participación de los docentes en proyectos de investigación, de tal manera que estas investigaciones contribuyan tanto con el enriquecimiento del currículo universitario, como con la articulación de la teoría y la práctica contable.

Como lo afirma Gómez (2012, p.6)

Enseñar y educar en contabilidad ya no basta, por el contrario, se necesita ir más allá, en el sentido de que quien enseña, quien educa y quienes reciben la acción de enseñar y de educar, deben propender por tener presente sobre quién recae la acción, quién es ese sujeto, para qué y por qué debe ejercerse la acción hacia ese sujeto, cuáles son las condiciones necesarias para que la acción se ejecute en óptimas condiciones de objetividad y subjetividad y por último, cuál es la realidad que se transforma durante el ejercicio de la acción. (...) Enseñabilidad y educabilidad, son procesos más que acciones que deben ejercerse en contabilidad, que deben pensarse y repensarse, de tal manera que posibiliten la transformación de los procesos educacionales que normalmente se desarrollan en los claustros universitarios (...) en el entendido de que dicha transformación parte de la comprensión crítica del mundo y que refiere una revisión de las ópticas positivistas que invaden el ejercicio educativo.

En función del argumento expuesto, resulta de interés enfatizar en la necesidad de replantear los modelos educativos tradicionales que han sustentado la enseñanza de la contabilidad, pues como se ha planteado en los párrafos anteriores, lo contable trasciende el plano de los conocimientos teóricos que sirven de base para la aplicación de las normas contables vigentes, lo cual implica la formación de profesionales competentes, para construir el conocimiento interdisciplinario, de carácter teórico y metodológico, requerido para aportar respuestas a los diversos problemas sociales vinculados con el área contable.

Tales modelos educativos deben articular adecuadamente las funciones de extensión, investigación y docencia universitaria, para así fomentar la construcción y utilización de métodos, técnicas e instrumentos, que permitan abordar la problemática contable de manera interdisciplinaria, interpretar críticamente los fenómenos con base en los referentes teóricos generados a partir de las investigaciones relacionadas con el objeto de estudio, incorporar dentro de las investigaciones las nuevas variables que abarca el hecho contable, así como los fenómenos del entorno que inciden en su funcionamiento.

Siguiendo a Zaá (2010, p.3) esto implica abandonar el excesivo objetivismo y las prácticas esquematistas y recetarias, para asumir que la realidad contable es tan compleja como los objetos de otras ciencias; y que tal realidad no solo se expresa mediante cuantificaciones, sino también a través de la narratividad científica donde el lenguaje de la contabilidad juega un papel protagónico.

Sobre la base de lo expuesto se deduce que la investigación en el ámbito contable debe renovarse y replantearse para considerar tanto las nuevas dinámicas de las disciplinas, como de las sociedades, pues como lo afirma Gómez (2013)

Si los contextos sociales se cimentan en las lógicas dialécticas que determinan las relaciones sociales y organizacionales, proponer soluciones a las problemáticas sociales, con base en las antiguas maneras de ver el mundo, limita el ejercicio propositivo de la contabilidad en pro de las problemáticas suscitadas.

Si los contextos sociales se cimentan en las lógicas dialécticas que determinan las relaciones sociales y organizacionales, proponer soluciones a las problemáticas sociales, con base en las antiguas maneras de ver el mundo, limita el ejercicio propositivo de la contabilidad en pro de las problemáticas suscitadas.

De esta manera, a partir del replanteamiento de la investigación contable, y de la incorporación de los 
desarrollos generados en el currículum universitario, los actores que intervienen en el proceso de enseñanza - aprendizaje alcanzarán una comprensión crítica de la realidad, pues precisarán los eventos, factores y elementos que contribuyen con la resolución de los problemas contables que demanda la sociedad y el entorno.

Tal replanteamiento como lo expone Gómez (2013) debe realizarse desde una posición interdisciplinaria, pues para enseñar la contabilidad en el nuevo contexto se requiere integrar los saberes contables con las propuestas de otras disciplinas, identificando de manera clara los aportes que estas últimas realizan a la ciencia contable.

Asimismo, esto conlleva a un replanteamiento de la educación contable para considerar el contexto en el que se desarrolla esta disciplina, en la búsqueda de que los futuros profesionales se encuentren en capacidad de responder tanto a las interrogantes relacionadas con la aplicación de la técnica y la norma contable, como a las vinculadas con el cómo y, el para qué, a partir de la construcción de un conocimiento socialmente pertinente, que permita integrar adecuadamente la teoría y la práctica contable.

En este contexto para una formación integral del contador público, se requiere la aplicación de algunas estrategias orientadas hacia: la investigación formativa para promover el proceso de construcción de conocimiento en el aula, el diagnóstico de la realidad social como fundamento para la discusión y el debate, así como la participación de los estudiantes y facilitadores en actividades extraacadémicas como eventos científicos, talleres, seminarios con los gremios y actores que aplican en la práctica la norma, entre otras.

\section{Conclusiones}

A partir de la revisión documental realizada para el desarrollo del estudio, se deduce la necesidad de aplicar nuevos enfoques en el campo de la investigación contable, pues en el contexto global se requiere de una práctica capaz de incidir en la teoría y viceversa, de modo que el proceder de los profesionales contables se deberá reorientar para conseguir avances significativos y socialmente pertinentes tanto en el saber, como en el hacer contable.

Evidentemente, en esta propuesta de articular la teoría y la práctica contable, existe un amplio campo para el desarrollo de propuestas de investigación, pues invita a una constante reflexión y crítica de los preceptos científicos formulados en contabilidad, así como a la evaluación de la aplicación de las normas en la práctica. Es decir, se requiere de un discurso que permita aproximar las bases epistemológicas de la disciplina contable al escenario aplicativo.

En este contexto, destaca el papel de la Universidad en la formación de profesionales contables con competencias para construir y transferir el conocimiento contable, que la sociedad actual y, las nuevas tendencias de investigación demandan. Para tal fin, se ha comenzado a instrumentar el modelo educativo basado en competencias para la formación de profesionales integrales, éticos, reflexivos, críticos y autónomos, con capacidad para articular la teoría y la práctica en la resolución de problemas. Tal modelo, se sustenta en la integración de los siguientes saberes: ser, hacer y conocer.

Finalmente, cabe mencionar que el International Accounting Standards Board (IASB) como organismo regulador de la práctica contable, en la búsqueda de reducir el distanciamiento entre el discurso teórico y la práctica contable, a través de la formulación de las Normas Internacionales de Información Financiera (NIIF), ha propuesto la armonización contable para adaptar la normativa que regula la práctica profesional en los distintos países, a las normas mencionadas, lo cual permite la utilización de un lenguaje estandarizado en la revelación e interpretación de la información financiera.

\section{Referencias}

Carr, W. \& Kemmis, S. (1988). Teoría crítica de la enseñanza. Barcelona: Martínez Roca.

Casal R. \& Viloria, N. (2007). La ciencia contable, su historia, filosofía, su evolución y su producto. Actualidad Contable (10) 15, 19-28. Recuperado de http:// 
www.saber.ula.ve/bitstream/123456789/17418/1/articulo2.pdf

Gómez, M. (2003). Contabilidad: Comentarios sobre Contabilidad: Comentarios sobre el discurso científico y los el discurso científico y los el discurso científico y los determinantes morales determinantes morales determinantes morales. Innovar, revista de ciencias administrativas y sociales (22), 109-120. Recuperado de http://www.scielo.org.co/pdf/inno/ v13n22/v13n22a10.pdf

Gómez, M. (2006). Comentarios sobre el aprendizaje - construcción de la teoría contable. Lumina (7), 129-153.Gómez, Y. (2012). Enseñabilidad y educabilidad de la contabilidad: primer acercamiento. XIII Asamblea ALAFEC. Universidad de Buenos Aires. Recuperado de http://www.alafec.unam.mx/memoria_xiii.php.

Gómez, Y. (2013). Enseñabilidad y educabilidad. Nuevas tendencias en la enseñanza y la investigación contables. Contexto, (1), 179-192. Recuperado de http://www.tdea.edu.co/images/noticias/documentos/revista_en_contexto/en_contexto_art8.pdf

González, L. (2005). Fundamentación conceptual de la disciplina contable. Revista internacional legis de contabilidad $\mathcal{E}$ auditoría, 151-192. Recuperado de http:// www.academia.edu/2900051/Fundamentaci\%C3\%B3n_conceptual_de_la_crisis_contable_-_Colciencias

Larrinaga, C. (1999). Perspectivas alternativas de investigación en contabilidad: una revisión. Revista de Contabilidad, 2(3), 103-131. Recuperado de http:// www.rc-sar.es/verPdf.php?articleId=45

Laughlin, L. (2011). Accounting Research, Policy and Practice: Worlds togetheror Worlds Apart? En: Evans, E.; Burritt, R.; Guthrie, J. (Editores). Bridging the Gap between Academic Accounting Research and Professional Practice (pp. 21-30). Australia: The Institute of Chartered Accountants in Australia, Centre for Accounting, Governance and Sustainability, University of South Australia.

Mattessich, R. (2003). Contabilidad: ¿cisma o síntesis? El desafío de la teoría condicional-normativa. Partida Doble, (144), 104-119. Recuperado de http:// pdfs.wke.es/4/7/0/2/pd0000014702.pdf

Ospina, C. (2005). Sobre la investigación en contabilidad algunos apuntes. PORIKAN, 73-121. Recuperado de http://www.unicauca.edu.co/porik_an/imagenes_ 3noanteriores/No.11porikan/articulo2.pdf

Tua, J. (1993). La enseñanza de la contabilidad en un contexto social. Quipukamayoc, 1(1), 34-50.

Tua, J. (1995). Evolución del concepto de contabilidad a través de sus definiciones. En Lecturas de Teoría e Investigación Contable (pp. 121-188). Recopilación de artículos del autor. Medellín: Centro Interamericano Jurídico-Financiero. Recuperado de http://www. academia.edu/11298865/EVOLUCION_DEL_CONCEPTO_DE_CONTABILIDAD_A_TRAVES_DE_ SUS_DEFINICIONES

Tucker, B. \& Parker, L. (2012). In our ivory towers? The research-practice gap in management accounting: an academic perspective. Recuperado de http://dx. doi.org/10.2139/ssrn.2130224

Viloria, N. (2013). Los retos de la contabilidad. Una visión desde los avances de la teoría contable. Lumina, (14),40-52. Recuperado de http://www.umanizales.edu.co/publicaciones/campos/economicas/ lumina/recursos/14/10.pdf

Zaá, J. (2010). Del discurso científico de la modernidad a una nueva narratividad en la construcción del conocimiento contable, Tendencias de la Investigación Contable. Primer Encuentro Internacional de Investigadores en Ciencias Contables organizado por la Universidad de Antioquia, el Politécnico Jaime Isaza Cadavid y la Universidad de los Andes (Venezuela), Medellín. 